\title{
A Methodology for Efficient Sketch Based Image Retrievals Based on Correlation Based Matching and Generalized Gamma Mixture Model (GGMM)
}

\author{
K M Vara Prasad, Ande Prasad
}

\begin{abstract}
The huge developments in touch screen devices and used in Computer vision developed the methodologies for recovering the images based on content. However in certain situations narration is the best suitable way to express and hence in the views of narration sketch based images are thus developed and utilized. These sketch based images are most useful in identifying face in criminal investigations. This paper provides a methodology for retrieving such sketch based images using the correlation based matching and generalized gamma mixture models. Performance is measured using precision and recall.

Keywords: Correlation Based Matching, Statistical Models, Criminal Investigations, Sketch-Based Images, Experimental Evaluation, Content Based Retrievals.
\end{abstract}

\section{INTRODUCTION}

The area of Object Based Image Retrieval is very useful in the area of image retrievals and the main advantage with this mechanism is that based on object effective retrievals from massive datasets are made possible. This object can have any features and can help in an identification of image under studying and thereby helping towards efficient retrievals[1][2].With these features many applications have been developed and are used in the domains of health care, medical, E-business, etc., [3][4][5]. Many models are thus subjected to using both parametric model based approaches and non parametric model based approaches. However in spite of the huge advantages of the Content Based retrieval mechanisms in particular situations where the people fail to express a query because of the ignorance of expression, effective retrievals cannot be possible. Therefore in such situation the concept Content Based Image Retrieval will fail not only this situation but also in most realistic scenarios where a witness at the crime incident tries to narrate the crime incident needs to have a more different approach. Recent trends for image recovery are focused in deep neural networks(DNN) that can generate better results at a high computational cost[6][7][8].Therefore to aid these situations sketch based images came into existence [9][10][11][12] and

Revised Manuscript Received on March 01, 2020.

* Correspondence Author

K M Vara Prasad*, Research Scholar, Department of Computer Email: varaprasad.kmv@gmail.com

Ande Prasad, Professor, Department of Computer Science, Vikrama Simhapuri University, Nellore (Andhra Pradesh), India. Email: prasadjkc@yahoo.co.in

(C) The Authors. Published by Blue Eyes Intelligence Engineering and Sciences Publication (BEIESP). This is an open access article under the CC BY-NC-ND license (http://creativecommons.org/licenses/by-nc-nd/4.0/) Science, Vikrama Simhapuri University, Nellore (Andhra Pradesh), India.

lot of applications are thus developed using of this application. However there is no methodology available in the literature that can identify the different styles of the artist because of the fact that each person poses his own artistic styles to express a particular scenario. Therefore to effectively identify such efficient scenarios statistical methodologies are well suited. With the advent of the depths convolutional networks, there has been an improvement in image recognition tasks in the image and sketch domain. Therefore in this paper a methodology presented considered a mechanism based on identification of sketches against the query using a correlation based matching technique where the images may be of different pose and orientations and these images compared using Generalized Gamma Mixture Model. The outputs derived are to be assessed for which different evaluation techniques such as precision, recalls are considered. The rest of the paper is presented in the following manner:

Section-2 provides the literature review of this paper. Section-3 provides the dataset considered for presenting the methodology. The section- 4 of the paper devoted towards the correlation based matching. Section-5 of the paper presents an overview of Generalized Gamma Mixture Model (GGMM). In section-6, the methodology for sketch based image retrieval is presented. The experimentation is highlighted and results derived are articulated in section-7 of the paper. The final section- 8 summarizes the article.

\section{REVIEW OF LITERATURE}

In this section of the article a detailed review about various articles presented by different authors and scientists is presented to showcase the status of a present work.

M Indu et al., (2016) published article in which the authors have tried to review the sketch based image retrieval methods. According to the authors, it is a complicated task to retrieve the facial objects from databases. In this article the authors have considered different types of Image Retrieval Systems like TBIR,CBIR, SBIR. In this work they have considered facial images in all the image databases. The extraction is based on PCA and Eigen faces. In this article the authors created face-sketch database for training data. This data is compared with the input sketch image to find the better matches. Apart from that PCA and Eigen values the authors used Multiscale Markov Random Field and KNDA for classification. The derived result provides good retrieval accuracy with minimum timestamp.

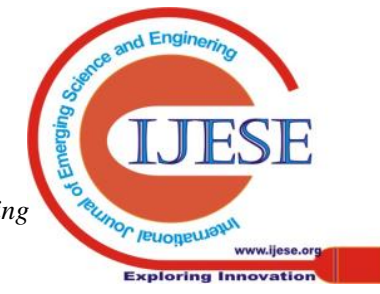


Shu Wang et al., (2015) published paper in which the authors have tried to retrieve Sketch-Based images Object Boundary Selection with HLR Descriptor. According to the authors, it is a complex task to reduce the gap between input sketch and photo-realistic images. In this article the authors have proposed line segment-based descriptor called as Histogram of Line Relationship (HLR). In this work they have considered a noise impact reduction algorithm called object boundary selection.GF-HOG features are also considered to identify the strokes within the sketch images. In this article, multiple hypotheses are generated for descriptors by hypothetical edge selection. Apart from that HLR and boundary selection, Bag-of-visual-words (BoVW) model is used. The proposed method provides better results.

Yonggang Qi et al., (2015) published article in which the authors have proposed better usage of edges via perceptual grouping. The authors can take this problem as two stage problem for grouping. Graph construction by ranking and portioning. They have used RankSVM to integrate two Gestalt principles, i.e., proximity and continuity. Then a multi-label graph cut algorithm is used to partition the graph, thus produces the edge grouping. Finally group filtering is used to generate final sketches. This method produced better results for segmentation of sketch-based images compared to state-of-the-art methods.

$\mathrm{Ke} \mathrm{Li} \mathrm{et} \mathrm{al.,} \mathrm{(2015)} \mathrm{published} \mathrm{article} \mathrm{in} \mathrm{which} \mathrm{the}$ authors have developed universal perceptual grouper for free hand sketches. This can be applied to any category of images. The authors were developed a novel deep perceptual grouping model learned with both generative and discriminative losses. They have used Gestalt principles like proximity and continuity. They have treated the sketch as sequence of strokes/segments, their model is sequence-to-sequence variational auto encoder. This grouper is useful for sketch semantic segmentation, synthesis and sketch based image retrieval. The proposed model performs better compared to the existing models.

Y Jhansi et al., (2015) published article in which the authors are proposed a method for effective retrieval of sketch based images based on Score level fusion. According to the authors, This system can extract features from the input query and sketches in the database and are compared efficiently. In this work the authors have used HOG and GMM features and these are combined with score level fusion. Apart from HOG and GMM, KL-divergence is used to compare two PDF's. This system used TU Berlin sketch data set with 20,000 sketches from 250 different objects. The average precision and recall values are better compared to individual HOG and GMM.

Ming-Ming Cheng et al., (2015) published article in which the authors have generated an algorithm for detecting salient regions based on global contrasting. The proposed algorithm produces high quality saliency maps. The proposed saliency maps are used for initializing a novel iterative version of GrabCut, called SaliencyCut, which is used for unsupervised salient object segmentation. The proposed algorithm produces top quality results on bench mark datasets. In this paper the authors have proposed Histogram-based contrast method to measure saliency and region-based contract is used provide spatial relations between pixels to segment into regions. In this article the authors have used GrabCut , which is an iterative process of graphcut and also used GMM for appearance mode. In this paper they used MSRA10K database provided by Liu et al. because it contains 10000 images with pixel-level labeling. Apart from SaliencyCut this algorithm is also used for sketch based image retrieval.This provides better results compared to the existing methods.

Sarthak Parui et al., (2015) published article in which the authors are proposed an approach for an image retrieval from millions of images on user sketched objects. According to the authors, the system can be invariant with translation, scale, rotation and small deformations. In this method each database image is preprocessed access the images faster in the online access. In this method, sequences of contour segments are used to extract shape information which are represented by variable length descriptors. In this article the authors performed chain similarities by using Dynamic Programming-based approximate matching algorithm. In this paper the authors used k-medoids based indexing to retrieve the databases in seconds. The authors tested this system with 1 million Flickr images from MIRFLICKR-1M and images from Imagenet database. This system provides better results compared to existing systems in case of large database images.

Xiaoqiang Ji et al., (2015) published article in which authors have tried to segmentation of cell images. According to authors the cell image segmentation is difficult because of its inaccurate cell images. In this article the authors tried to provide effective approach watershed algorithm for fine segmentation. The authors followed better approach to get fine segmentation, i.e enhance the image, OSTU threshold segmentation and watershed algorithm for fine segmentation. Ana Maria Miguelena Bada et al., (2014) published paper in which the authors have tried to generate Sketch-Based Image Retrieval System for Web .This system is introduced as an alternative to text-based image search on web. According to the authors, Existing systems face the challenge regarding edge detection for content based image retrieval for larger regions and avoiding textures. In this article the authors have developed an evolutionary algorithm which optimizes the image processing parameters. In this article a canny algorithm is used to extract connected and thin edges. Apart from that mean-shift segmentation is also used. This method will provide better results with low volume database.

Houssen Chatbri et al., (2014) published article in which the authors have tried to spot the query image in document images. According to the authors, it is a complex task due to noise and complexity of document images. In this article the authors have developed a modular spotting algorithm. This algorithm is segmentation -free and application-independent. In this work they have considered general images. In this article a robust spotting is developed in two stages. First one is modular framework based on pruning and voting detecting the occurrence of query and second one is optimization using Genetic Algorithm. Apart from that Genetic Algorithm PSO-based optimization scheme is also used. The derived result provided good retrieval accuracy with minimum timestamp.

Dibya Jyothi Bora et al.,(2014) published an article in which authors proposed a novel approach towards clustering based segmentation. In this paper k-means clustering algorithm is used for segmenting color images. In this paper the authors used the cosine distance measure. 
The author generated the following sequence for image segmentation: k-means algorithm with cosine distance measure, sobel filter for filtering, marker watershed algorithm. MSE(Mean Squared Error) and PSNR(Peak Signal to Noise Ratio) are used as performance metrics. The authors used Matlab demo images for their work. This novel approach provides better results for simple color images.

N Raghu Ram Reddy et al., (2014) published paper in which the authors have generated Color Sketch-Based Image Retrieval using color and texture features. According to the authors, it is a complex task to reduce the gap between color input sketch and photo-realistic images. In this article the authors have combined color and texture features to compare the color sketch and image. In this work they have extracted color features using HSV(Hue, Saturation, Value) color space and texture features are extracted using Gray-Level Co-occurrence Matrix(GLCM). In this article the authors compared the features of sketch and image in the database using Euclidean distance metric. This article uses the Wang database for experimental purpose. The proposed method provides better retrieval accuracy compared to the SBIR.

\section{DATASET CONSIDERED}

In order to present the proposed methodology, a real time data set is developed and is utilized for the experimentation purpose. Every image is preprocessed and it is normalized to a fixed size of $150 X 150$.these image comprises of sketch views generated by different artist with different strokes and curvatures. The sample data set is depicted in Fig.1 of the article.

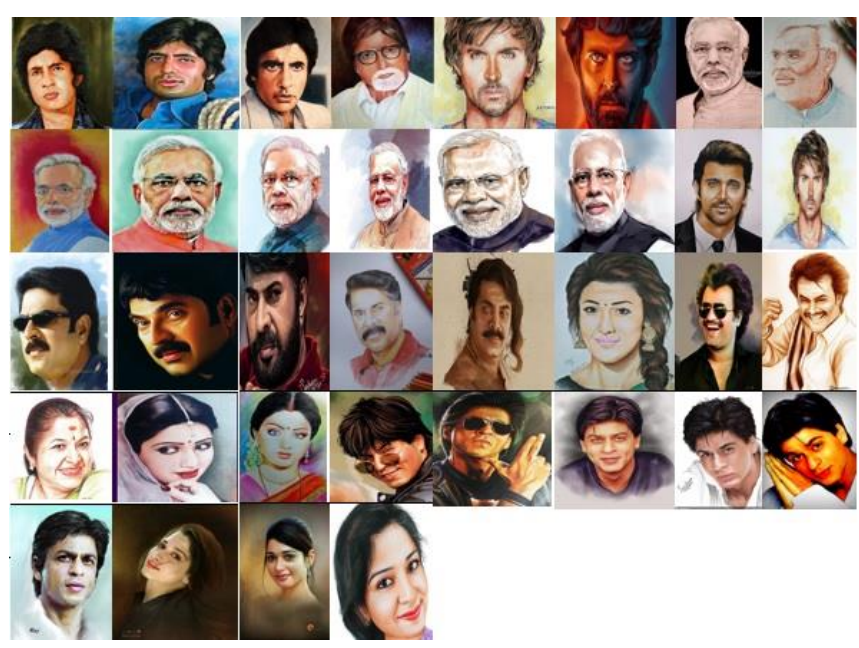

Fig. 1.Sample data set.

\section{CORRELATION BASED MATCHING}

The proposed model is presented for the usage at remote areas, by considering the input to sketch based images of the patients. These to sketch based images are to be searched for relevancy among the related to sketch based images from the image databases collected and thereby the treatment suggested by the experts can be gathered. This information plays a vital role while delivering suggestions and primary treatment to the patients at remote areas. Since the treatment/suggestion is completely based on similarity, the relevancy is to be estimated accurately. In this thesis we present a novel methodology for identifying the relevancy between the sketch based images in the database and feature or a query image is considered for relevance. An image correlation is utilized for establishing the relevancy. The main advantage of this methodology is that, it can be used to test even if a sub-part of the image is given as input. The formulas for the comparing the similarity are given by

$$
C(L, K)=\frac{\sum_{i=1}^{n} L * K}{\sqrt{\operatorname{sum}\left(L_{1} \cdot L_{2}\right)} * \operatorname{sum}\left(K_{1} \cdot K_{2}\right)}
$$

Where $\mathrm{L}$ is the image from the database and $\mathrm{K}$ is the query image (Note: $\mathrm{K}$ and $\mathrm{L}$ should be of same dimension) and we say that the query image $\mathrm{K}$ is the part of the image $\mathrm{L}$, if the correlation approaches to near 1.

\section{GENERALIZED GAMMA DISTRIBUTION}

The probability density function of generalized gamma distribution is given by

$$
f(x, k, c, a, b)=\frac{c(x-a)^{c k-1} e^{-\left(\frac{x-a}{b}\right)^{c}}}{b^{c k} \Gamma(k)}
$$

Where a, b, c, k is called the Gamma variants and c, k is called shape parameters such that $\mathrm{c}, \mathrm{k}>0$. a is called location parameter, $\mathrm{b}$ is called shape parameter with $\mathrm{a}, \mathrm{b}>0$.

The mean of the Generalized Gamma Distribution is given by

$$
\frac{a+b \Gamma\left(c+\frac{1}{\mathrm{k}}\right)}{\Gamma(\mathrm{c})}
$$

The variance of the Generalized Gamma Distribution is given by

$$
\left.b^{2} \frac{\Gamma\left(\mathrm{c}+\frac{2}{\mathrm{k}}\right)}{\Gamma(\mathrm{c})}-\left\{\Gamma\left(\mathrm{c}+\frac{1}{\mathrm{k}}\right) / \Gamma(\mathrm{c})\right]^{2}\right\}
$$

The mode of Generalized Gamma Distribution is given by

$$
a+b\left(c-\frac{1}{k}\right)^{\frac{1}{k}}, c>\frac{1}{k}
$$

The rth moment about the Location parameter ' $a$ ' is given by

$$
b^{k}(\Gamma(\mathrm{c}+\mathrm{r} / \mathrm{k}) /(\Gamma(\mathrm{c}))
$$

We assume that every image as a k-component Generalized Gamma distribution and its Probability Density Function is of the form

$$
h(x)=\sum_{i=1}^{k} \alpha_{i} g_{i}\left(y_{i} \mid \mu_{i}, \sigma_{i}^{2}, \lambda\right)
$$

Here, $\lambda$ is the skewness parameter and $\mathrm{k}$ is the number of regions, $\alpha_{i}>0$ are weights such that $\sum_{i=1}^{k} \alpha_{i}=1$ and

$$
f(x, k, c, a, b)=\frac{c(x-a)^{c k-1} e^{-\left(\frac{x-a}{b}\right)^{c}}}{b^{c k} \Gamma(k)}
$$

$\alpha \_i$ is the probability of occurrence of the ith component of the Generalized Gamma Distribution, i.e., the probability of the ith image sample. Generally, it can be taken as the ratio of the size of the ith pixel to the size of the entire image data such that,

$$
\sum_{i=1}^{k} \alpha_{i}=1
$$

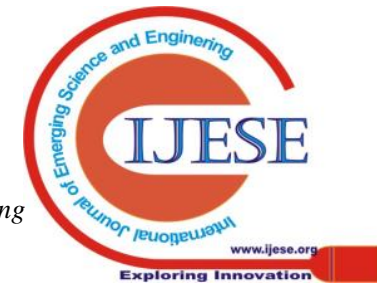




\section{SKETCH BASED IMAGE RETRIEVAL ALGORITHM}

Step 1: Obtain histogram of all the to sketch based images in the database, in order to model the various particular cases of Generalized Gamma Distribution.

Step 2: Apply Principal Component Analysis method Step 3: Cluster the image using Fuzzy C-Means Algorithm. Step 4: Calculate Probability Density Function (PDF) using Generalized Gamma Distribution.

Step 5: Find the relevant image based on correlation using the above formula 1.

\section{EXPERMENTATION AND RESULTS}

The experimentation is conducted in Dot NET environment considering the real-time generated dataset containing different sketch based images, for the experimentation with Dataset. Among this dataset we have considered 150 sketches data as test data.

In order to experiment the model proposed, we have considered the data set and the features are extracted using principal component analysis and clustered using fuzzy C-means algorithm and the classification is carried out using GGMM. Relevant images are obtained by using correlation. The various inputs and outputs are as follows:

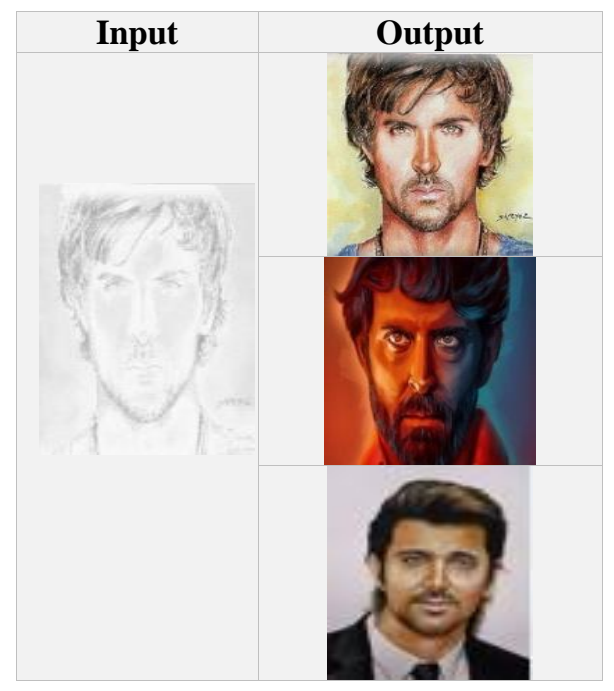

Fig. 2.: Input and Output images.

In order to evaluate the model various performance metrics such as Precision, Recall, Error Rate and Retieval Efficiency are used. DCT is used as a feature vector for all the metrics. The following table shows various performance metrics for real-time dataset.

Table- I: Performance Metrics

\begin{tabular}{|c|c|l|c|c|c|c|}
\hline $\mathbf{R}$ & $\mathbf{N R}$ & $\mathbf{R} / \mathbf{N R}$ & Precision & Recall & $\begin{array}{c}\text { Error } \\
\text { Rate }\end{array}$ & $\begin{array}{c}\text { Retrieval } \\
\text { Efficiency }\end{array}$ \\
\hline 120 & 750 & 0.16 & 84 & 12 & 9 & 84 \\
\hline 98 & 700 & 0.14 & 77 & 29 & 16 & 77 \\
\hline 78 & 650 & 0.12 & 65 & 38 & 28 & 70 \\
\hline 60 & 600 & 0.1 & 56 & 45 & 30 & 55 \\
\hline 44 & 550 & 0.08 & 45 & 56 & 48 & 49 \\
\hline 30 & 500 & 0.06 & 38 & 65 & 55 & 48 \\
\hline 18 & 450 & 0.04 & 29 & 77 & 64 & 55 \\
\hline 8 & 400 & 0.02 & 9 & 84 & 74 & 56 \\
\hline
\end{tabular}

$R:$ No. of relevant to sketch based images.

NR: No. of non-relevant to sketch based images.
R/NR: Ratio of relevant to non-relevant.

The graphical representation of the performance metrics is shown in below figure.

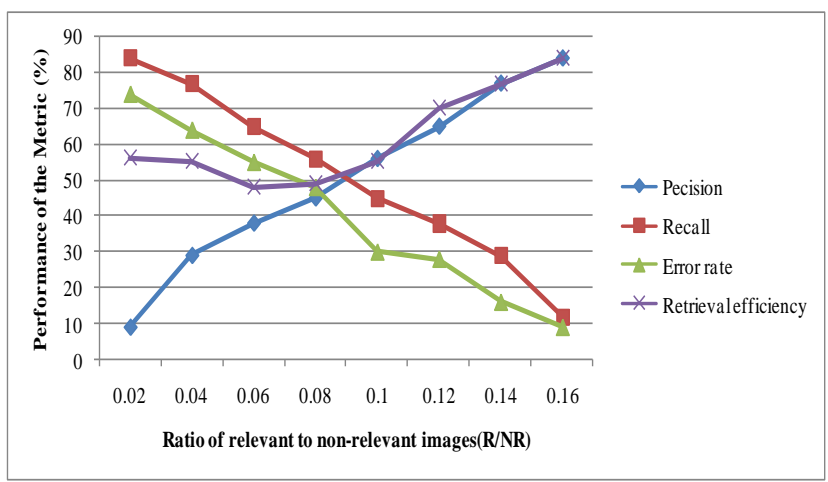

Fig. 3.Comparision of all the metrics.

\section{CONCLUSION}

In this paper a methodology is presented to retrieve the image based on sketches. In order to extract the features, principle component analysis is used and matching is performed based on correlation. The Query image is posed and based on the relevance, most relevant to sketch based images are extracted. The classification is carried out using GGMM algorithm. The performance accuracy is carried out using various metrics like Precision, Recall, Error Rate and Retrieval Efficiency. The results developed showcase that the developed methodology could provide better retrieval accuracy. This method can be well suited for application of cyber forensic and criminal investigations.

\section{REFERENCES}

1. M Indu and Kavitha K V,"Survey on sketch based image retrieval methods",ICCPCT,2016.

2. Shu Wang, Jian Zhang, Tony X. Han, and Zhenjiang Miao,"Sketch-Based Image Retrieval Through Hypothesis-Driven Object Boundary Selection With HLR Descriptor", IEEE TRANSACTIONS ON MULTIMEDIA, VOL. 17, NO. 7, JULY 2015.

3. Yonggang Qi,Yi-Zhe Song,Tao Xiang,Honggang Zhang,Timothy Hospedales,Yi Li,Jun Guo,"Making Better Use of Edges via Perceptual Grouping",IEEE,2015.

4. Ke Li,Kaiyue Pang.Yi-Zhe Song,Tao Xiang,Timothy $M$. Hospedales,and Honggang Zhang," Towards Deep Universal Sketch Perceptual Grouper", IEEE Transactions on Image Processing, 2019.

5. Y. Jhansi,E. Sreenivasa Reddy," A Methodology for Sketch based Image Retrieval based on Score level Fusion",International Journal of Computer Applications, Volume 109 - No. 3, 2015.

6. Ming-Ming Cheng,Niloy J.Mitra,Xiaolei Huang,Philip H.S.Torr,and Shi-Min Hu,"Global Contrast Based Salient Region Detection",IEEE,2015.

7. Sarthak Parui and Anurag Mittal,"Similarity-invariant Sketch-based Image Retrieval in Large Databases",European Conference on Computer vision,2014.

8. Xiaoqiang Ji, Yang Li, Jiezhang Cheng, Yuanhua Yu,and MeijiaoWang,"Cell Image Segmentation Based on an Improved Watershed Algorithm",ICISP,2015.

9. Ana María Miguelena Bada, Guillermo de Jesús Hoyos Rivera, Antonio Marín Hernández, "Garabato: A proposal of a Sketch-Based Image Retrieval System for the Web",IEEE,2014.

10. Houssem Chatbri, Paul Kwan, and Keisuke Kameyama," A Modular Approach for Query Spotting in Document Images and Its Optimization Using Genetic Algorithms", IEEE Congress on Evolutionary Computation (CEC), 2014 
11. Dibya Jyoti Bora and Anil Kumar Gupta,"A Novel Approach Towards Clustering Based Image Segmentation",IJESE,2014.

12. N Raghu Ram Reddy,G Suresh Reddy,and M Narayana,"Color Sketch Based Image Retrieval",IJAREEIE,2014.

\section{AUTHORS PROFILE}

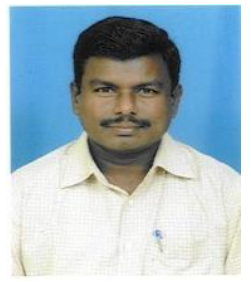

Mr. K. M. Vara Prasad, had obtained his B.Tech and M.Tech in Computer Science and Engineering from Acharya Nagarjuna University, Guntur, Andhra Pradesh, India. He has teaching experience of 10 Years in the department of Computer Science and Engineering. Currently he is perusing Ph.D in Digital Image Processing. His research areas of interest are Digital Image Processing, Artificial Intelligence, Pattern Recognition, and Data Mining.

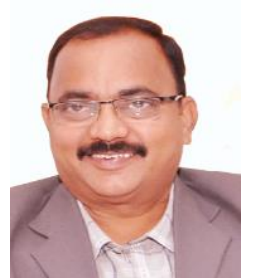

Dr. Ande Prasad, received his M.Sc. (Mathematics), M.Phil. (Engineering Mathematics), M.Tech. (Computer Science \& Technology), Ph.D (Computer Science \& Systems Engineering) from Andhra University, Visakhapatnam, Andhra Pradesh, India. He is working as professor in computer science department, vikrama simhapuri university and has more than 23 years of enriched experience in teaching, research and administration. He worked in various capacities viz Co-ordinator, Head, Vice-Principal, Chairmen and Member (UG / PG): Board of Studies in Computer Science / Computer Science and Engineering, Officer In-charge: College Information Cell, Member: Technical Advisory Committee etc. He is as Supervisor/ Co-supervisor to guide Ph.D.'s and is currently guiding six Ph.D. Full-Time and Part-Time scholars. He has more than 50 publications in International and National Journals and Conferences with good impact factor and reckoning. His research insights were presented in Conferences, Seminars and Symposia both on National and on the International platforms. His research areas of interests are Speech Processing, Pattern Recognition, Image Processing, Cloud Computing and Computer Networks etc. 